\title{
Influence of Medial Wedge Support on Pain and Function in Patients with Patellofemoral Pain Syndrome
}

\author{
MOSTAFA I. EL-SHAFEY, M.Sc.*; NADIA A. FAYAZ, Ph.D.**; EBTESSAM F. GOMAA, Ph.D.** and \\ AHMED H. HAFEZ, M.D.*** \\ The Department of Physical Therapy for Musculoskeletal Disorders, Faculty of Physical Therapy, \\ Delta University for Science \& Technology* and Cairo University** and The Department of Orthopaedic Surgery, \\ Faculty of Medicine, Ain Shams University***, Egypt
}

\begin{abstract}
Background: Patellofemoral Pain Syndrome (PFPS) is a common complaint in athletes and populations which described as a dull and aching pain in anterior or retropatellar area in absence of other pathology. It is commonly believed that main problems of PFPS are pain and also impaired knee function.

Aim of Study: To investigate the efficacy of medial wedge support on pain relief and functional improvement in patients with PFPS.

Material and Methods: Thirty patients (10 females and 20 males) aged between 18-35 years were assigned randomly into two equal groups. Experimental group (A): Included 15 patients were treated with hip abductors and lateral rotators strengthening exercises in addition to wearing medial wedge support whereas control group (B): Included 15 patients were treated with only hip abductors and lateral rotators strengthening exercises. Anterior knee function was measured by Anterior Knee Pain Scale (AKPS) and pain intensity was measured by Visual Analogue Scale (VAS) in both groups before and after 12 successive sessions over six weeks.

Results: The findings of this study indicated a significant decrease in VAS and significant improvement in AKPS after treatment in comparison to before treatment values in both groups A and B. Post-treatment comparison of both groups showed statistically significant difference with better improvement in group A and $p=0.0018$ for AKPS and $p=0.0004$ for VAS.

Conclusion: The obtained results suggest that using medial wedge support together with the hip abductors and lateral rotators strengthening exercises have proved to be more beneficial in decreasing pain intensity and improving functional level in Patellofemoral Pain Syndrome (PFPS) patients.
\end{abstract}

Key Words: Medial wedge support - Patellofemoral pain syndrome - Visual analogue scale-Anterior knee pain scale.

Correspondence to: Dr. Mostafa I. El-Shafey, E-Mail:pt.mostafa@yahoo.com

\section{Introduction}

PATELLOFEMORAL Pain Syndrome (PFPS) is a common complaint in athletes and populations especially in which repetitive lower limb loading is involved, with reported incidence rates in physically active young adult greater than $25 \%$ [1] Proximal factors including hip muscle weakness have been proposed to contribute to patellofemoral malalignment and the development of PFPS $[2,3]$ Multiple studies have established a correlation between hip external rotator and abductor weakness in women with PFPS $[4,5]$. Observational studies have demonstrated an association between excessive foot pronation and PFPS [6]. During gait, it is thought that a more pronated foot type leads to excessive or prolonged pronation of the foot and therefore greater tibial and femoral internal rotation [7]

It is generally agreed that PFPS should be managed initially by conservative rather than surgical [8] . Conservative treatment include taping, strengthening of the quadriceps muscle, flexibility training, biofeedback, manual therapy to the lower quadrant, and fitting of foot orthoses [9-12].

Recent studies have reported significant impairments in hip abduction and lateral rotation strength which suggested that strengthening exercises for these hip muscles could be as important factor in managing patellofemoral pain by improving the control of hip adduction and internal rotation moments during weight-bearing activities $[\mathbf{1 , 1 3}]$.

It is thought that foot orthoses may reduce pain by limiting foot pronation and associated lower limb rotation, thereby reducing lateral Patellofemoral Joint (PFJ) forces [14]. 
Clinical trials have shown that exercising the hip muscles or using foot orthoses are efficacious in managing PFPS [15-17] but to the researcher knowledge, there is no study investigating the combined effect of foot orthoses with hip abductors and lateral rotators strengthening exercises on pain intensity and functional level in treatment of patients with PFPS.

Therefore, this study was investigating the combined effect of medial wedge support with strengthening hip abductors and lateral rotators exercises on pain intensity and functional level in patients with PFPS.

\section{Patients and Methods}

This study was conducted in the out-patient clinic of the Faculty of Physical Therapy, Delta University for Science and Technology during 2017. The study was conducted on thirty patients (10 females and 20 males). The patients were assigned randomly into two equal groups. Experimental group (A): 15 patients with mean of age $(24.0 \pm 5.16$ years $)$ and mean BMI $(24.67 \pm 2.56$ $\mathrm{kg} / \mathrm{m}^{2}$ ) were treated by hip abductors and lateral rotators strengthening exercises in addition to wearing medial wedge support whereas control group (B): 15 patients with mean of age $(22.0 \pm$ 1.60 years $)$ and mean BMI $\left(23.07 \pm 2.88 \mathrm{~kg} / \mathrm{m}^{2}\right)$ were treated with only hip abductors and lateral rotators strengthening exercises. This study was conducted in the out-patient clinic of the Faculty of Physical Therapy, Delta University for Science and Technology in the period from August 2017 to January 2018. Patients in both groups participated in the study for 6 weeks, two sessions per week. The ethical committee number is: 012/ 001750 .

\section{Procedures:}

\section{A-Assessment procedures:}

I- Knee functional assessment procedures:

The functional performance was assessed using AKPS [18]. The Arabic AKPS is sufficiently reliable, valid, and appropriate for use as a patient reported outcome measure for Arabic speaking individuals with anterior knee pain and PFPS. It is also the first validated knee outcome measure in Arabic to assess knee pathology [19]

\section{II- Pain intensity assessment procedures:}

Pain was assessed by using Visual Analogue Scale (VAS) which is a $10 \mathrm{~cm}$ calibrated line with 0 (zero) representing no pain and 10 representing worst pain. The examiner illustrated the meaning of the VAS to the patient, and the patient was asked to make a mark at the point which represents his pain. The distance between zero and the mark was then measured and recorded in $\mathrm{mm}$ [20].

\section{B- Treatment procedures:}

1- Prefabricated medial wedge support: The medial wedge support was commercially available three-quarter-length devices with lateral cutouts (flat foot support, manufactured in China) and imported by (El-Shafey Company, Kafr El-Sheikh, Egypt). It is made of ethelene-vinyl acetate of medium density, containing built-in arch supports and $4^{\circ}$ varus rear foot wedging. No customization of the orthoses was performed. However, size was individualized, ensuring that the first metatarsophalangeal joint was just distal to end of the orthoses. Participants were asked to wear footwear that was able to accommodate the orthoses whenever possible during daily living activities [21].

\section{2- Strengthening exercises:}

A- Hip abductors strengthening exercise: The patient lied in side lying position on the nonaffected side. The patient was asked to bend the knee of non-affected side and extend the affected knee. The therapist took a stride position behind the patient and stabilized the pelvis. A sandbag was wrapped just proximal to lateral malleolus and the patient was asked to raise his limb in abduction, hold for 6 seconds count, then return to starting position and relax [22].

$B$ - Hip lateral rotators strengthening exercise: The patient sit at the edge of the plinth with the hip and knee flexed to 90 degree, and his hand behind him for support. The therapist took a stride position beside the patient on the affected side and stabilized the patient's thigh. Sandbag was wrapped just proximal to lateral malleolus, the patient was asked to rotate the leg inward toward the nonaffected side for 6 seconds count, then return to starting position and relax [21]

The sandbag weighed from $(2 \mathrm{Kg}-10 \mathrm{Kg})$. Each strengthening exercise of hip was performed for 2 sets of 10 repetitions with 3 seconds rest between repetitions for 2 sets and 1 minute rest after each set [21]. Each patient was trained at $60 \%$ of $10-$ Repetition Maximum (RM) (the amount of weight that could be lifted and lowered through available range of motion exactly 10 times) [23].

The two groups received two sessions per week, for six successive weeks. 


\section{Results}

All analyses were carried out using a statistical software program (GraphPad Prism Version 5.0; GraphPad Software, San Diego, CA, USA). Prior to final analysis, data were tested for normality test and homogeneity of variance. Descriptive statistics included mean and standard deviation was used for all variables. Intra group comparison of the pre-interventional and post- interventional outcome measures were done by using a paired $t$ test. Inter group comparison of the pre-interventional and post-interventional outcome measures were done by using unpaired $t$-test.

\section{Baseline and demographic data:}

In the present study, there was non-significant $(p \geq 0.05)$ difference in the age and body dimensions between experimental and control patients. The mean of age was $24.0 \pm 5.16$ and $22.0 \pm 1.60$ for experimental and control patients respectively. The mean of BMI was $(24.67 \pm 2.56$ vs. $23.07 \pm 2.88)$ for experimental and control patients respectively.

\section{The effect on the Anterior Knee Pain Scale (AKPS):}

As presented in (Table 1), during pre-treatment AKPS was similar $(p=0.60)$ between experimental and control groups. Post-treatment AKPS was extremely greater $(p=0.0018)$ in experimental than control groups. Moreover, within the same group either experimental or control there was a highly significant $(p<0.001)$ difference of AKPS between pre and post-treatment.

Table (1): The effect on AKPS (mean \pm STD) with normality test (in parentheses).

\begin{tabular}{|c|c|c|c|c|}
\hline \multirow{2}{*}{$\begin{array}{l}\text { Patient } \\
\text { group }\end{array}$} & \multirow{2}{*}{$\begin{array}{r}\text { No. of } \\
\text { patients }\end{array}$} & \multicolumn{2}{|c|}{ AKPS } & \multirow{2}{*}{$\begin{array}{c}p- \\
\text { value }\end{array}$} \\
\hline & & $\begin{array}{c}\text { Pre- } \\
\text { treatment }\end{array}$ & $\begin{array}{l}\text { Post- } \\
\text { treatment }\end{array}$ & \\
\hline $\begin{array}{l}\text { Experimental } \\
\text { Normality test }\end{array}$ & 15 & $\begin{array}{l}73.07 \pm 9.26 \\
(0.17)\end{array}$ & $\begin{array}{l}95.67 \pm 3.95 \\
(0.08)\end{array}$ & $p=0.000$ \\
\hline $\begin{array}{l}\text { Control } \\
\text { Normality test }\end{array}$ & 15 & $\begin{array}{l}71.20 \pm 10.21 \\
(0.42)\end{array}$ & $\begin{array}{l}90.47 \pm 4.30 \\
(0.64)\end{array}$ & $p=0.000$ \\
\hline$p$-value & - & 0.60 & 0.0018 & - \\
\hline
\end{tabular}

\section{Visual analogue scale:}

As shown in (Table 2), there was non-significant difference in VAS between experimental and control groups' pre-treatment. Post-treatment VAS was extremely $(p=0.0004)$ different between experimental and control groups. Within the same patients group either experimental or control there was highly significant $(p<0.0001)$ difference between pre-treatment and post-treatment.
Table (2): The effect on the Visual Analogue Scale (VAS) for experimental and control patients.

\begin{tabular}{|c|c|c|c|c|}
\hline \multirow{2}{*}{$\begin{array}{l}\text { Patient } \\
\text { group }\end{array}$} & \multirow{2}{*}{$\begin{array}{l}\text { No. of } \\
\text { patients }\end{array}$} & \multicolumn{2}{|c|}{ Pain } & \multirow{2}{*}{$\begin{array}{c}p- \\
\text { value }\end{array}$} \\
\hline & & $\begin{array}{c}\text { Pre- } \\
\text { treatment }\end{array}$ & $\begin{array}{c}\text { Post- } \\
\text { treatment }\end{array}$ & \\
\hline $\begin{array}{l}\text { Experimental } \\
\text { Normality test }\end{array}$ & 15 & $\begin{array}{l}5.66 \pm 1.25 \\
(0.48)\end{array}$ & $\begin{array}{l}0.20 \pm 0.29 \\
(0.005)\end{array}$ & $p=0.000$ \\
\hline $\begin{array}{l}\text { Control } \\
\text { Normality test }\end{array}$ & 15 & $\begin{array}{l}5.23 \pm 1.23 \\
(0.89)\end{array}$ & $\begin{array}{l}0.96 \pm 0.68 \\
(0.60)\end{array}$ & $p=0.000$ \\
\hline$p$-value & - & 0.35 & 0.0004 & - \\
\hline
\end{tabular}

\section{Discussion}

Patellofemoral Pain Syndrome (PFPS) is a common complaint in athletes and populations [1] It is described as a dull and aching pain in anterior or retropatellar area in absence of other pathology [24].

Hip muscles weakness especially hip abductor and lateral rotators weakness $[3,25]$ and excessive foot pronation have been associated with patellofemoral malalignment and thereby PFPS [26,27]

Clinical trials had shown that hip abductors and lateral rotators strengthening exercises [22,28] or medial wedge support were efficient in managing PFPS [14,29].

This study was conducted to investigate the combined effect of medial wedge support with strengthening hip abductors and lateral rotators exercises on pain intensity and functional level in PFPS patients.

In comparison between the two groups, the results obtained in the current study showed high significant difference between the two groups $(p=0.0004)$ for the mean of VAS and significant difference between the two groups $(p=0.0018)$ for the mean of Kujala patellofemoral scoring system (AKPS).

These findings were supported by many researches as following:

In a pilot study of 20 adolescent females, it was suggested that the addition of medial wedge support to an exercise program resulted in significantly greater improvements in the level of pain measured by VAS than treatment with flat insoles and exercises over eight weeks [30].

It was showed that a combination of medial wedge support with activity modification program had led to significant improvement in pain intensity on VAS. However, the inclusion criteria used in this study was neutral rear-foot alignment in 
subtalar joint, forefoot-to-rear-foot alignment, navicular drop, relaxed calcaneal stance and Qangle [31]

In a randomized clinical trial, it was stated that adding multimodal physiotherapy which included joint mobilization, patellar taping, quadriceps muscle training and PFPS education to medial wedge support may enhance overall clinical success in the pain intensity measured by VAS and functional level measured by AKPS. However, it had been found that medial wedge support only provided greater short to medium term (6 weeks) improvements over flat inserts in AKPS and no significant difference was found when compared with the physiotherapy program [32].

The present study agree with a cohort study which found that the medial wedge support showed immediate improvements in the patients' functional task performance and pain level measured by AKPS and VAS sequentially especially in the pronated foot patients. It explained that as the medial wedge support prevented excess foot pronation and thereby knee valgus which enabled patients to perform functional tasks with less pain [6].

In a systematic review, it was concluded that medial wedge support produced positive patient outcomes as a stand-alone treatment or when combined with physiotherapy treatment and clinicians should consider their use when treating individuals with PFPS based on limited evidence [33]

The literature on foot orthotics suggested a role for prefabricated medial wedge supports combined with physiotherapy in reducing pain and improving functionality in specific types of patients with PFPS [34].

In another follow-up study, it was found that 14 patients reported improvements in pain and functional levels measured by AKPS and VAS sequentially after 12 weeks of using medial wedge support. However, there were some limitations in this study as there was no control group and did not incorporate standard strengthening or physiotherapy methods into the patients' treatment plans [20].

Also a prospective cohort study found improvement in AKPS achieved at 12 weeks after using prefabricated medial wedge support in 60 patients with PFPS [35].

It had been showed that prefabricated medial wedge support provided improvements in PFPS than a wait-and-see approach in terms of worst and usual pain reduction on VAS and functional improvement on AKPS [36]

It was stated that supplementation of strengthening of hip abductor and lateral rotator muscles in a strengthening quadriceps exercise program provided additional benefits with respect to the perceived pain symptoms measured with VAS during functional activities in patients with patellofemoral pain syndrome after six weeks of treatment [37].

It was stated that more pain reduction on VAS was associated with patients treated with hip exercises program including hip abductors and lateral rotators strengthening exercises combined with knee extensors strengthening exercises than knee extensors exercises alone [38].

It was found that after a 3-week hip abductor muscles strengthening exercises, there was a reduction in pain intensity on VAS in patients with PFPS [39]

It was found that an 8-week program of hip abductors and lateral rotators strengthening exercises combined with core muscles strengthening exercises had an improvement in pain intensity and functional level on VAS and AKPS respectively [40].

It was stated that targeting hip abductors and lateral rotators strengthening exercises initially may be more efficient, allowing for muscle training while reducing exacerbation of patellofemoral symptoms. The patients who started with hip strengthening reported an earlier and more significant drop in knee pain on VAS after only 4 weeks of rehabilitation, while the patients who initially performed quadriceps strengthening required 8 weeks of rehabilitation to achieve a similar decrease in pain [41].

Also it was found that an isolated 8-week hip abductors and lateral rotators strengthening exercises program was effective in improving pain on VAS in females with PFPS compared to nonexercise controlled group [16]

It was stated that knee stretching and strengthening exercises supplemented by hip posterolateral musculature strengthening exercises which included (hip abductor, lateral rotator and extensor muscles) were more effective than knee exercises alone in improving long-term function on AKPS in sedentary women with PFPS [42]

In a randomized clinical trial, the patients who used knee strengthening exercises in addition with 
hip abductors and lateral rotators strengthening exercises had shown improvement in functional level on AKPS and reduction in pain intensity on VAS more efficiently than knee strengthening exercises alone [21].

In another study, it was suggested that additional hip strengthening exercises with Closed Kinetic Chain (CKC) program of knee and hip exercises has the advantage of more pain relief on VAS and functional improvement on AKPS than CKC exercises alone [43].

This is also supported by a study which stated that a 6-week hip strengthening protocol resulted in earlier resolution of pain on VAS compared with knee strengthening protocol. However, the hip protocol included hip extensors and internal rotators strengthening exercises [44].

Finally from the previous discussion of these results and according to reports of other investigators in similar studies, it can explained that the combined effect of medial wedge support with hip abductors and lateral rotators strengthening exercises in group (A) had significantly improvements on pain intensity and functional level on VAS and AKPS respectively than hip abductors and lateral rotators strengthening exercises alone in group (B). So, medial wedge support had shown to be effective only in reducing the symptoms of PFPS by reduction in internal tibial rotation which produced a concurrent reduction in internal femoral rotation and thereby a reduction in lateral directed Patellofemoral Joint (PFJ) forces and altered PFJ contact pressures leading to improvements in pain intensity and functional level [7].

\section{Conclusion:}

Based on the findings of the current study, it is concluded that using medial wedge support together with the hip abductors and lateral rotators strengthening exercises have proved to be more beneficial than using the hip abductors and lateral rotators strengthening exercises alone, owing to the significant improvements in pain intensity and functional level in patients with Patellofemoral Pain Syndrome (PFPS).

\section{References}

1- ROBINSON R.L. and NEE R.J.: Analysis of hip strength in females seeking physical therapy treatment for unilateral patellofemoral pain syndrome. Journal of Orthopaedic \& Sports Physical Therapy, 37 (5): 232-8, 2007.

2- FULKERSON J.P.: Diagnosis and treatment of patients with patellofemoral pain. The American Journal of Sports Medicine, 30 (3): 447-56, 2002.
3- SOUZA R.B. and POWERS C.M.: Differences in hip kinematics, muscle strength, and muscle activation between subjects with and without patellofemoral pain. Journal of Orthopaedic \& Sports Physical Therapy, 39 (1): 12-9, 2009.

4- BOLGLA L.A., MALONE T.R., UMBERGER B.R. and UHL T.L.: Hip strength and hip and knee kinematics during stair descent in females with and without patellofemoral pain syndrome. Journal of Orthopaedic \& Sports Physical Therapy, 38 (1): 12-8, 2008.

5- IRELAND M.L., WILLSON J.D., BALLANTYNE B.T. and DAVIS I.M.: Hip strength in females with and without patellofemoral pain. Journal of Orthopaedic \& Sports Physical Therapy, 33 (11): 671-6, 2003.

6- BARTON C.J., BONANNO D., LEVINGER P. and MENZ H.B.: Foot and ankle characteristics in patellofemoral pain syndrome: A case control and reliability study. Journal of Orthopaedic \& Sports Physical Therapy, 40 (5): 28696, 2010.

7- POWERS C.M.: The influence of altered lower-extremity kinematics on patellofemoral joint dysfunction: A theoretical perspective. Journal of Orthopaedic \& Sports Physical Therapy, 33 (11): 639-46, 2003.

8- DURSUN N., DURSUN E. and KILIÇ Z.: Electromyographic biofeedback-controlled exercise versus conservative care for patellofemoral pain syndrome. Archives of physical medicine and rehabilitation, 82 (12): 1692-5, 2001.

9- CROSSLEY K., BENNELL K., GREEN S. and McCONNELL J.: A systematic review of physical interventions for patellofemoral pain syndrome. Clinical Journal of Sport Medicine, 11 (2): 103-10, 2001.

10- YIP S.L. and NG G.Y.: Biofeedback supplementation to physiotherapy exercise programme for rehabilitation of patellofemoral pain syndrome: A randomized controlled pilot study. Clinical rehabilitation, 20 (12): 1050-7, 2006.

11- PEELER J. and ANDERSON J.E.: Effectiveness of static quadriceps stretching in individuals with patellofemoral joint pain. Clinical Journal of Sport Medicine, 17 (4): 234-41, 2007.

12-IVERSON C.A., SUTLIVE T.G., CROWELL M.S., MORRELL R.L., PERKINS M.W., GARBER M.B., MOORE J.H. and WAINNER R.S.: Lumbopelvic manipulation for the treatment of patients with patellofemoral pain syndrome: Development of a clinical prediction rule. Journal of Orthopaedic \& Sports Physical Therapy, 38 (6): 297 312,2008

13- CICHANOWSKI H.R., SCHMITT J.S., JOHNSON R.J. and NIEMUTH P.E.: Hip strength in collegiate female athletes with patellofemoral pain. Medicine \& Science in Sports \& Exercise, 39 (8): 1227-32, 2007.

14- GROSS M.T. and FOXWORTH J.L.: The role of foot orthoses as an intervention for patellofemoral pain. Journal of Orthopaedic \& Sports Physical Therapy, 33 (11): 66170, 2003.

15- COLLINS N., CROSSLEY K., BELLER E., DARNELL R., McPOIL T. and VICENZINO B.: Foot orthoses and physiotherapy in the treatment of patellofemoral pain syndrome: Randomised clinical trial. B.M.J., 337, a1735, 2008. 
16- MILLS K., BLANCH P., DEV P., MARTIN M. and VICENZINO B.: A randomised control trial of short term efficacy of in-shoe foot orthoses compared with a wait and see policy for anterior knee pain and the role of foot mobility. Br. J. Sports Med., 46 (4): 247-52, 2012.

17- KHAYAMBASHI K., MOHAMMADKHANI Z., GHAZNAVI K., LYLE M.A. and POWERS C.M.: The effects of isolated hip abductor and external rotator muscle strengthening on pain, health status, and hip strength in females with patellofemoral pain: A randomized controlled trial. Journal of Orthopaedic \& Sports Physical Therapy, 42 (1): 22-9, 2012.

18- KUJALA U.M., JAAKKOLA L.H., KOSKINEN S.K., TAIMELA S., HURME M. and NELIMARKKA O.: Scoring of patellofe-moral disorders. Arthroscopy: The Journal of Arthroscopic \& Related Surgery, 9 (2): 159-63, 1993.

19- ALSHEHRI A., LOHMAN E., DAHER N.S., BAHIJRI K., ALGHAMDI A., ALTORAIRI N., ARNOS A. and MATAR A.: Crosscultural adapta-tion and psychometric properties testing of the Arabic anterior knee pain scale. Medical Science Monitor: International Medical Journal of Experimental and Clinical Research, 23: 1559, 2017.

20- JENSEN M.P., CHEN C. and BRUGGER A.M.: Interpretation of visual analog scale ratings and change scores: A reanalysis of two clinical trials of post-operative pain. The Journal of Pain, 4 (7): 407-14, 2003.

21- BARTON C.J., MENZ H.B. and CROSSLEY K.M.: The immediate effects of foot orthoses on functional performance in individuals with patellofemoral pain syndrome. British Journal of Sports Medicine, 45 (3): 193-7, 2011.

22- MOHAMED K.A.H.: Influence of strengthening of hip abductors and external rotators in treatment of patellofemoral pain syndrome. (An Unpublished M.Sc theses, Cairo University) Egypt, 2012.

23- KISNER C. and COLBY L.A.: Therapeutic exercise: Foundations and techniques. Philadelphia, PA: FA Davis Co, 66-104, 2007.

24- LOWRY C.D., CLELAND J.A. and DYKE K.: Management of patients with patellofemoral pain syndrome using a multimodal approach: A case series. Journal of Orthopaedic \& Sports Physical Therapy, 38 (11): 691-702, 2008.

25- FERBER R., DAVIS I.M. and WILLIAMS III D.S.: Gender differences in lower extremity mechanics during running. Clinical biomechanics, 18 (4): 350-7, 2003.

26- TANG S.F., CHEN C.K., HSU R., CHOU S.W., HONG W.H. and LEW H.L.: Vastus medialis obliquus and vastus lateralis activity in open and closed kinetic chain exercises in patients with patellofemoral pain syndrome: An electromyographic study. Archives of Physical Medicine and Rehabilitation, 82 (10): 1441-5, 2001.

27- PIVA S.R., FITZGERALD K., IRRGANG J.J., JONES S., HANDO B.R., BROWDER D.A. and CHILDS J.D.: Reliability of measures of impairments associated with patellofemoral pain syndrome. B.M.C. musculoskeletal disorders, 7 (1): 33, 2006.

28- BOLING M.C., PADUA D.A. and ALEXANDER CREIGHTON R.: Concentric and eccentric torque of the hip musculature in individuals with and without patellofemoral pain. Journal of Athletic Training, 44 (1): 7-13, 2009.
29- McCONNELL J.: The physical therapist's approach to patellofemoral disorders. Clinics in Sports Medicine, 21 (3): 363-87, 2002.

30- ENG J.J. and PIERRYNOWSKI M.R.: Evaluation of soft foot orthotics in the treatment of patellofemoral pain syndrome. Physical Therapy, 73 (2): 62-8, 1993.

31- SUTLIVE T.G., MITCHELL S.D., MAXFIELD S.N., McLEAN C.L., NEUMANN J.C., SWIECKI C.R. and FLYNN T.W.: Identification of individuals with patellofemoral pain whose symptoms improved after a combined program of foot orthosis use and modified activity: A preliminary investigation. Physical therapy, 84 (1): 4961, 2004.

32- COLLINS N., CROSSLEY K., BELLER E., DARNELL R., McPOIL T. and VICENZINO B.: Foot orthoses and physiotherapy in the treatment of patellofemoral pain syndrome: Randomised clinical trial. B.M.J., 337, a1735, 2008.

33- BARTON C.J., MUNTEANU S.E., MENZ H.B. and CROSSLEY K.M.: The efficacy of foot orthoses in the treatment of individuals with patellofemoral pain syndrome. Sports Medicine, 40 (5): 377-95, 2010.

34- MUNUERA P.V. and MAZOTERAS-PARDO R.: Benefits of custom-made foot orthoses in treating patellofemoral pain. Prosthetics and orthotics international, 35 (4): 342 9, 2011.

35- BARTON C.J., MENZ H.B. and CROSSLEY K.M.: Effects of prefabricated foot orthoses on pain and function in individuals with patellofemoral pain syndrome: A cohort study. Physical therapy in sport, 12 (2): 70-5, 2011.

36- MILLS K., BLANCH P., DEV P., MARTIN M. and VICENZINO B.: A randomised control trial of short term efficacy of in-shoe foot orthoses compared with a wait and see policy for anterior knee pain and the role of foot mobility. Br. J. Sports Med., 46 (4): 247-52, 2012.

37- NAKAGAWA T.H., MUNIZ T.B., BALDON R.D.M., DIAS MACIEL C., De MENEZES REIFF R.B. and SERRÃO F.V.: The effect of additional strengthening of hip abductor and lateral rotator muscles in patellofemoral pain syndrome: A randomized controlled pilot study. Clinical rehabilitation, 22 (12): 1051-60, 2008.

38- RAZEGHI M., ETEMADI Y., TAGHIZADEH S. and GHAEM H.: Could hip and knee muscle strengthening alter the pain intensity in patellofemoral pain syndrome? Iranian Red Crescent Medical Journal, 12 (2): 104, 2010.

39- FERBER R., KENDALL K.D. and FARR L.: Changes in knee biomechanics after a hip-abductor strengthening protocol for runners with patellofemoral pain syndrome. Journal of Athletic Training, 46 (2): 142-9, 2011.

40- EARL J.E. and HOCH A.Z.: A proximal strengthening program improves pain, function, and biomechanics in women with patellofemoral pain syndrome. The American Journal of Sports Medicine, 39 (1): 154-63, 2011.

41- DOLAK K.L., SILKMAN C., MCKEON J.M., HOSEY R.G., LATTERMANN C. and UHL T.L.: Hip strengthening prior to functional exercises reduces pain sooner than quadriceps strengthening in females with patellofemoral pain syndrome: A randomized clinical trial. Journal of Orthopaedic \& Sports Physical Therapy, 41 (8): 560-70, 2011. 
42- FUKUDA T.Y., MELO W.P., ZAFFALON B.M., ROSSETTO F.M., MAGALHÃES E., BRYK F.F. and MARTIN R.L.: Hip posterolateral musculature strengthening in sedentary women with patellofemoral pain syndrome: A randomized controlled clinical trial with 1-year followup. Journal of Orthopaedic \& Sports Physical Therapy, 42 (10): 823-30, 2012.

43- ISMAIL M.M., GAMALELDEIN M.H. and HASSA K.A.: Closed kinetic chain exercises with or without additional hip strengthening exercises in management of patellofemoral pain syndrome: A randomized controlled trial. European Journal of Physical and Rehabilitation Medicine, 49 (5): 687-98, 2013.

44- FERBER R., BOLGLA L., EARL-BOEHM J.E., EMERY C. and HAMSTRA-WRIGHT K.: Strengthening of the hip and core versus knee muscles for the treatment of patellofemoral pain: A multicenter randomized controlled trial. Journal of Athletic Training, 50 (4): 366-77, 2015.

\section{تآثير الدعامة الوتدية الإنسية على متتلازمة آلم آسفل الفخذ الفخد والرضفة}

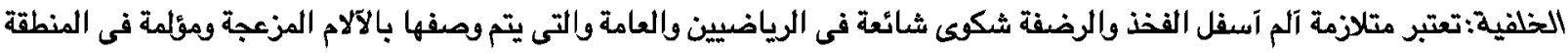

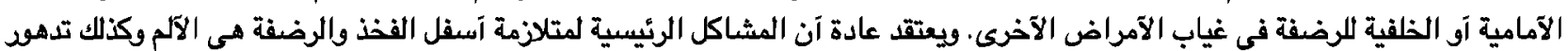

هدف الدراسة: دراسة فاعلية الدعامة الوتدية الإنسية على الآلم والآداء الوظيفى فى مرضى متلازمة آسفل الفخذ والرضفة.

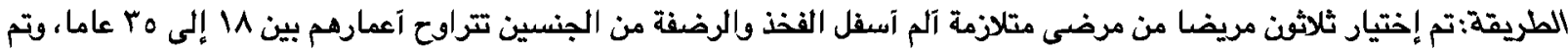

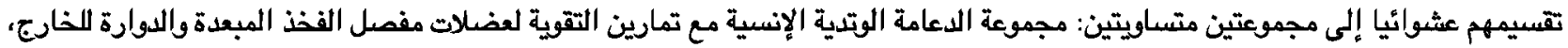

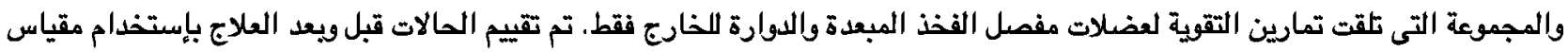

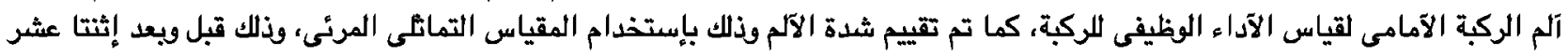
جلسة من العلاج فى خلال ست آسابيع.

النتائج: آظهرت نتائج البحث وجود فروق ذات دلالة إحصائية عالية للمجموعتين عند مقارنة نتائج ما بعد العلاج بنتائج ما قبل العلاج. كما

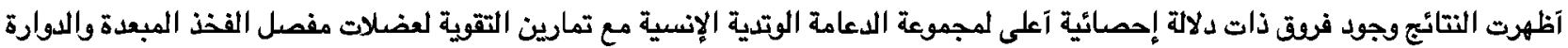

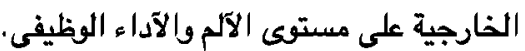

الإستتاج: من التتائج السابق عرضها فإن إستعمال الدعامة الوتدية الإنسية مع تمارين التقوية لعضلات مفصل الفخذ المبعدة والدوارة

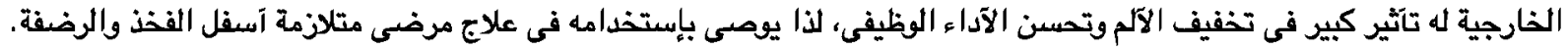

